\title{
One versus two adjacent interdigital neuroma excision: a patient outcome study
}

\author{
Kefilwe Boineelo Benjamin' ${ }^{\mathbb{D}}$, Nikiforos Saragas ${ }^{1,2}$ (D), Paulo Ferrão $0^{1,2}$ (D) \\ 1. University of Witswatersrand. \\ 2. Netcare Linksfield Hospital.
}

\begin{abstract}
Objective: We aimed to evaluate patient satisfaction after surgery for both single and two adjacent neuromas.

Methods: We reviewed the data of patients treated operatively for interdigital neuromas between 2003 and 2016 . We interviewed them and administered the Self-Reported Foot and Ankle Score questionnaire. Patient scores were then analyzed categorically, and variation between groups was assessed.

Results: Sixty-two patients were available for review. Thirty-one patients had a single interdigital neuroma excised and 31 had two adjacent interdigital neuromas excised. Twenty-eight of the 31 (90\%) patients with a single neuroma had good or excellent results while 23 (74.2\%) of those with adjacent neuromas had similar outcomes. One patient with a single neuroma had a poor result while four patients with adjacent neuromas had poor results. The mean score was 41 (excellent) for patients with a single interdigital neuroma and 37 (good) for those with adjacent neuromas $(\mathrm{p}=0.473)$. The majority of patients in both groups would undergo surgery again.

Conclusion: We found no statistically significant difference in outcomes of patients who undergo surgery for either single or two adjacent interdigital neuromas. General patient satisfaction is good and/or excellent post excision.
\end{abstract}

Level of Evidence II; Prognostic Studies; Retrospective Study.

Keywords: Foot; Morton neuroma; Neuroma; Patient satisfaction.

\section{Introduction}

The clinical symptoms of interdigital neuroma, also known by the eponym Morton's neuroma, were first described by Civinni $^{(1)}$ in 1835, Durlacher(2) in 1845, Coughlin and Pinsonneault in $2001^{(3)}$. In 1876, Morton(4) reported on a case series of patients with similar symptoms. However, he attributed the symptoms to trauma/injury to the fourth metatarsophalangeal joint.

Interdigital neuroma is now known to be a benign condition of the interdigital plantar nerve. Clinically, patients present with pain in the forefoot, typically radiating to the toes and aggravated by wearing tight shoes ${ }^{(5)}$. It commonly affects the third web space, followed by the second web space, and rarely occurs in the first and fourth web spaces. The common digital nerve in the third web space, formed by branches of the lateral and medial plantar nerve, is thought to be relatively thicker and more tethered in the web space, making it more susceptible to microtrauma. In addition, high-heeled shoes with a narrow toe box can cause compression of the metatarsal heads and push the common digital nerve up against the unyielding transverse intermetatarsal ligament ${ }^{(6-8)}$.

An interdigital neuroma is a tumor-like mass of perineural, epineural, and endoneural fibrosis with loss of myelinated nerve fibers ${ }^{(9,10)}$. The diagnosis of interdigital neuroma is mainly a clinical one ${ }^{(5,11)}$. Pastides et al. ${ }^{(5)}$ reported a $98 \%$ sensitivity based on history and clinical examination alone. They recommended the use of magnetic resonance imaging (MRI) or ultrasound (US) to identify multiple neuromas or localize the web space when it is clinically unclear. Sobiesk et al. ${ }^{(12)}$ assessed the effectiveness of US to accurately identify interdigital neuromas and reported that it can predict the presence, size, and location of neuromas with a $100 \%$ sensitivity and an $83.3 \%$ specificity. US is therefore a cost-effective, noninvasive modality that can be used for equivocal cases
Study performed at the Linksfield Private Hospital, Johannesburg, Gauteng, South Africa.

Correspondence: Kefilwe Boineelo Benjamin. University of Witswatersrand, 7 York Road, Parktown - 2198, Johannesburg, South Africa. E-mail: kbenjamin@bohss.co.bw. Conflicts of interest: none. Source of funding: none. Date received: October 2, 2021. Date accepted: October 22, 2021. Online: December 20, 2021.

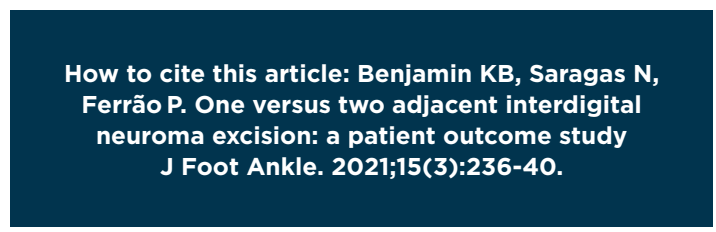


of neuroma to confirm the diagnosis. The management of interdigital neuromas includes both conservative and operative treatment. Conservative therapies include accommodative footwear, anti-inflammatory medications, innersoles, and physiotherapy ${ }^{(9,13-15)}$. In addition, local injection of corticosteroids, sclerosing agents, and local anesthetic has been found to provide short-lived relief of symptoms. Surgery is usually reserved for intractable symptoms despite optimal conservative therapy. Outcome studies of operative treatment of neuromas generally address patients with a single neuroma. Most of these studies have reported satisfactory outcomes following operative management of single interdigital neuro$\operatorname{mas}^{(3,6,7,11,16,17)}$. To our knowledge, only one study looked primarily at the clinical results of patients who had more than one neuroma excised ${ }^{(18)}$.

The existence of multiple interdigital neuromas in the same foot has been reported as rare by some authors; however, Valero et al. ${ }^{(17)}$ recently showed a $65.2 \%$ prevalence of multiple neuromas in the same foot. Benedetti et al. ${ }^{(18)}$ evaluated 19 feet with simultaneous adjacent neuromas, which were managed surgically, and reported outcomes that were comparable to those reported by other authors for single neuromas ${ }^{(17)}$. To our knowledge, no other studies in the published English medical literature have addressed this since Benedetti et al. No studies have directly compared outcomes in patients with single versus adjacent neuromas.

Our study reviewed a series of patients who underwent surgery by a single surgeon, and we investigated their self-reported satisfaction after surgery for both single and adjacent neuromas. The hypothesis of this study was that simultaneous excision of adjacent neuromas would yield poorer outcomes compared to single neuroma excision.

\section{Methods}

Ethics approval was obtained from the University of Witwatersrand ethics and scientific committee (M171067).

The primary aim of this study was to assess the outcomes of surgery undertaken for interdigital neuroma. Secondarily, we compared the outcomes in patients with a single neuroma versus those with adjacent neuromas in the same foot. We reviewed the data of patients managed surgically for interdigital neuromas by a single surgeon between January 2003 and January 2016. We included all adult patients who had undergone surgery for either single or adjacent interdigital neuromas. Patients with concomitant foot disorders or those who had other surgery to the foot in question were excluded. All patients had undergone preoperative US investigation, which confirmed the presence of either single or double neuromas in the foot. The US was performed by a sonographer with 21 years of musculoskeletal experience using a $12 \mathrm{MHz}$ linear-array transducer. A hypoechoic mass, which could be identified in the longitudinal plane and on dynamic studies during side-to-side compression of the forefoot, was considered diagnostic of a Morton's neuroma (Figure 1).
The surgery was performed by the senior author. All operations were performed under general anesthesia with a tourniquet. No nerve blocks were used. A dorsal incision was made either over the second or third web space for excision of single neuromas, or the third metatarsal for excision of two adjacent neuromas to allow access to both the second and third web spaces. The deep transverse ligament was then transected. Each neuroma was then excised (Figure 2). All specimens were sent for histology, and analyses confirmed them to be interdigital neuromas. The wounds were closed using subcuticular sutures. Postoperatively, patients were placed in a padded dressing and kept heel walking as tolerated for two weeks.

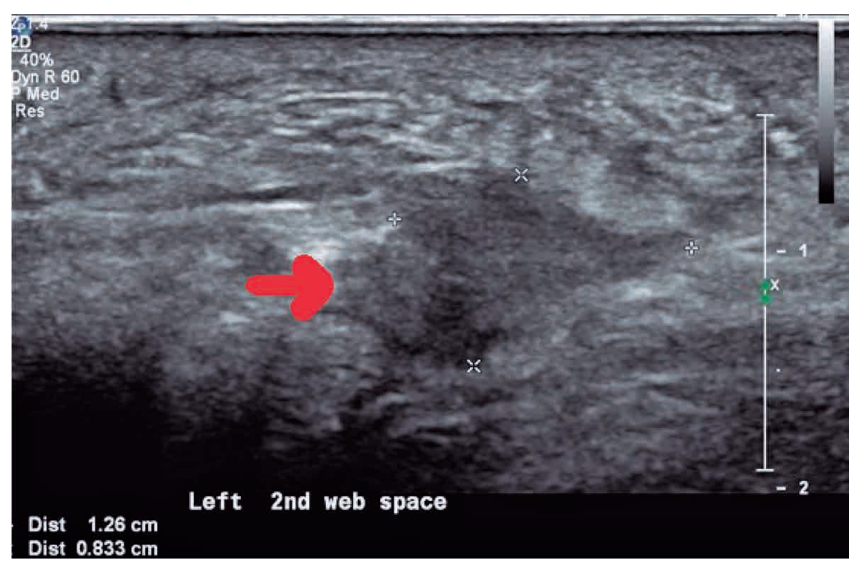

Figure 1. Red arrow shows a hypoechoic mass seen on ultrasound suggestive of interdigital neuroma.

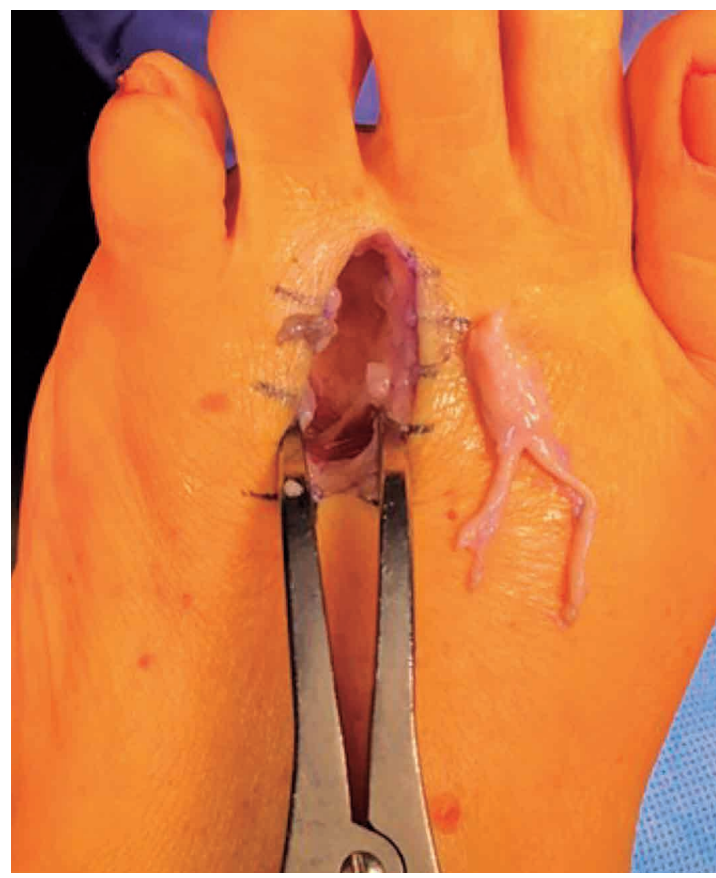

Figure 2. Image shows interdigital neuroma post excision from the third web space. 
All patients were contacted independently by one of the authors, who was not involved with the surgeries. Patients were asked to complete the validated Self-Reported Foot and Ankle Score (SEFAS). The SEFAS questionnaire has good psychometric properties for evaluation of patients with different disorders and operative procedures around the forefoot, hindfoot, and ankle joint ${ }^{(19,20)}$. If a patient had bilateral operations, the worst foot was scored. A total SEFAS score of $O$ represents the most severe disability while a score of 48 represents normal function. A score less than 20 was graded as poor, 20-29 as average, 30-39 as good, and 40-48 as excellent. The results were grouped categorically based on whether the scores were poor, average, good, or excellent. An analysis of variance (ANOVA) test was then carried out to assess significance in the variation seen between the two groups of patients. Statistical significance was defined at $5 \%(p \leq 0.05)$.

\section{Results}

The total number of neuromas was 114 in 62 patients. Thirty-one patients had adjacent second and third web-space neuromas in the same foot, with 12 patients having bilateral foot involvement. Of the 31 patients with a single neuroma, three had bilateral foot involvement (Table 1). Twenty-eight patients had unilateral involvement, 20 of whom had third web-space neuromas and 8 had second web-space neuromas. The follow-up time for single neuromas ranged from 0.6 to 13.6 years, with a mean follow-up of 6.2 years. For adjacent web-space neuromas, follow-up ranged from 0.5 to 11.2 years, with a mean follow-up of 4.6 years.

In patients with adjacent web-space neuromas, 23 (74.2\%) had excellent or good outcome scores. Of these, 18 (58\%) had excellent outcome scores and 5 (16\%) had good outcome scores. Four (12.9\%) had average outcomes, and four (12.9\%) had poor outcomes. Of those with poor outcomes, one patient reported moderate pain while the other three reported severe pain affecting them most days and limiting their activities of daily living. Of the 31 patients, 24 (77.4\%) said they would have surgery again. One patient, despite a poor functional score, said he would still have surgery again (Table 2).

For the patients with single neuromas, 28 (90\%) had excellent or good outcome scores. Twenty-one (67.7\%) of these patients had excellent outcome scores while seven (22.6\%) had good scores. Two (6.4\%) patients had average scores while only one (3.2\%) patient had a poor outcome score. The patient with the poor outcome complained of persistent pain and swelling in the foot, which felt similar to the neuroma pain he had prior to surgery (Table 2). Twenty-seven (87\%) patients said they would have the procedure done again. Surprisingly, three of the four patients who said they would not repeat the procedure had either good or excellent scores. The reason for declining a repeat operation included pain felt in the immediate postoperative period and bothersome numbness of the foot postoperatively.
Table 1. Patient demographics stratified according to whether they had single or adjacent neuromas $(n=62)$. The table further shows the proportion of patients who would repeat the procedure in the single vs. double adjacent neuroma groups

\begin{tabular}{lcc} 
& Single $(\mathbf{n = 3 1})$ & Adjacent $(\mathbf{n}=\mathbf{3 1})$ \\
\hline Female $(n=50)$ & 23 & 27 \\
Male $(n=12)$ & 8 & 4 \\
Mean age & 58.4 years $(34-74)$ & 55.1 years (41-71) \\
Unilateral & 28 & 19 \\
$\quad$ Left & 13 & 4 \\
$\quad$ Right & 15 & 15 \\
Bilateral & 3 & 12 \\
Number of patients who & & \\
would have surgery again & & $24(77 \%)$ \\
Yes & $27(87 \%)$ & $7(23 \%)$ \\
No & $4(13 \%)$ & \\
\hline
\end{tabular}

Table 2. Results stratified according to the Self-Reported Foot and Ankle Score (SEFAS): scores less than 20 were regarded as poor, 20-29 as average, 30-39 as good, and 40-48 as excellent $(n=62)$

\begin{tabular}{lccc|} 
Groups & $\begin{array}{c}\text { Single neuroma } \\
(\mathbf{n}=\mathbf{3 1})\end{array}$ & $\begin{array}{c}\text { Adjacent neuroma } \\
\mathbf{( n = 3 1 )}\end{array}$ & $\begin{array}{c}\text { Differences between } \\
\mathbf{(} \mathbf{p}=\mathbf{0 . 0 5})\end{array}$ \\
\hline Excellent & $21(67.7 \%)$ & $18(58.1 \%)$ & 0.473 \\
Good & $7(22.6 \%)$ & $5(16.1 \%)$ & 0.866 \\
Average & $2(6.5 \%)$ & $4(12.9 \%)$ & 0.448 \\
Poor & $1(3.2 \%)$ & $4(12.9 \%)$ & 0.814 \\
\hline
\end{tabular}

Among the patients with bilateral operations but single neuromas on either side, two (66.7\%) of the three had excellent outcomes while one (33.3\%) reported good function. However, among the patients with bilateral surgery and adjacent neuromas, only five (56\%) of the nine had excellent or good results while two (22\%) had average outcomes and two (22\%) had poor outcomes.

The mean outcome score was 41 (excellent) for the patients with single neuromas and 37 (good) for those with adjacent neuromas. However, this difference was not statistically significant $(p=0.13)$. While there was no statistical difference between groups for patients who had poor outcomes in either group, there was only one patient from the single neuroma group versus four from the adjacent neuroma group $(p=0.81)$. In subgroup analyses, the mean score for patients who had excellent scores was 46 in the single neuroma group and 45 in the adjacent neuroma group, with no statistically significant difference $(p=0.47)$. Overall, there was no difference between involvement of the right, left, or both feet in the single neuroma group $(p=0.600)$ ) or the adjacent neuroma group $(p=0.153)$. In addition, there was no difference in outcomes between men and women within each group (single group $\mathrm{p}=0.829$, adjacent group $\mathrm{p}=0.879$ ). 


\section{Discussion}

An interdigital neuroma is a common affliction with well-documented clinical presentation, diagnostic work-up, and management. Operative management is a well-recognized treatment option, and thus a study to evaluate outcomes of this common procedure is warranted. This study showed comparably good outcomes post excision of the interdigital neuroma(s) and no difference between patients with single or adjacent neuromas. Of the patients who reported poorer outcomes, the majority complained of troublesome numbness and difficulty with uneven surfaces as the causes of their dissatisfaction. In addition, some patients reported unbearable pain in the early postoperative period. Nonetheless, most patients in either group said they would repeat the procedure. In addition, our study has shown that the existence of simultaneous adjacent neuromas is more common than previously reported.

As far as we know, this study is one of the very few outcome studies addressing adjacent interdigital neuromas. Despite the well-documented demographics and management options, long-term outcomes post excision of the interdigital neuroma have rarely been reported ${ }^{(3,18,21-23)}$. Coughlin et al. ${ }^{(3)}$ reported $85 \%$ excellent or good results in their patients post excision. They also reported that adjacent neuromas or bilateralism did confer lower scores. Kasparek and Schneider ${ }^{(21)}$ reported $75 \%$ good or excellent results in their review of patients post excision of interdigital neuromas. However, they reported a significantly worse outcome in patients who had multiple neuromas excised $(p=0.038)$. Bucknall et al. ${ }^{(22)}$ also reported no statistical significance between single and adjacent neuroma excision using clinical scores. Their follow-up was only 6 months, patient-reported outcomes were not compared, and patients with concomitant foot surgery were not excluded. In contrast, Reichert et al. ${ }^{(23)}$ reported that patients who had single neuroma excision did significantly better than those who had adjacent neuroma excision. However, the adjacent neuroma group only had 8 patients compared to 33 patients in the single neuroma group. Our cohort had 31 patients in both the single and adjacent neuroma groups.

In a smaller series $(n=19)$, Benedetti et al. ${ }^{(18)}$ reviewed clinical results of patients with simultaneous adjacent interdigital neuromas and reported outcomes comparable to those of the single interdigital neuroma literature. They reported that $84 \%$ of their patients had acceptable results. In our case series, $74 \%$ of patients with adjacent neuromas had excellent or good outcomes post excision. The existence of adja- cent neuromas has been a subject of skepticism. Valero et al. ${ }^{(17)}$ recently reported on the incidence of multiple Morton neuromas in the foot. In 279 feet, they found an incidence of $65.2 \%$ for adjacent neuromas. They concluded that multiple neuromas in the same foot should not be regarded as a rare condition and in fact suggested that the presence of one neuroma should prompt the search for another. All our patients underwent US examination prior to surgery to assess adjacent neuromas, and all specimens taken at the time of surgery were sent for histology. In all cases, the histological examination confirmed the diagnosis of interdigital neuroma. Our study confirms that the existence of simultaneous adjacent neuromas is more common than previously reported. The authors recommend preoperative US examination by a musculoskeletal sonographer in suspected interdigital neuroma cases to confirm the diagnosis, identify the affected web space, and look for adjacent neuromas.

This study was a prospective analysis of retrospective data, which is a potential limitation. We used a well-documented, validated SEFAS questionnaire to assess patient satisfaction. In addition, we excluded patients with concomitant foot disorders or those who had additional procedures done at the time of surgery to remove any potential confounders. Our hypothesis was disproved, as there was no statistical difference in patient-reported outcomes between single versus adjacent neuroma excision.

\section{Conclusion}

This study was a long-term follow-up of patient outcomes following excision of either single or adjacent neuromas. Our review of 62 patients demonstrated no statistical difference in outcomes between groups. In our case series, patients with single interdigital neuromas had excellent or good outcomes post excision. Similar to the previously mentioned studies, patients with adjacent neuromas had lower outcome scores in our study. Despite the difference not being statistically significant, we suggest advising patients with adjacent neuromas of a slightly lower success rate post excision. In addition, gender, side of lesion, and bilateral foot involvement showed no difference in outcomes. Dissatisfaction was generally derived from postoperative pain or long-term numbness post excision, which made navigating uneven surfaces difficult. Overall, most patients had excellent or good results and would recommend the surgery. More studies, particularly prospective randomized controlled studies, are required to further elucidate this area of interest.

Authors' contributions: Each author contributed individually and significantly to the development of this article: KBB* (https://orcid.org/OOOO-OOO3-O2831329) Collected data, interpreted results, wrote the paper and reviewed successive versions; NS* (https://orcid.org/0000-0002-5566-7588) Conceived and planned the activities that led to the study, reviewed successive versions, and approved the final version; PF* (https://orcid.org/O000-0003-4639-0326) Participated in the interpretation of results, review process, and made significant contributions and approval of the final version. All authors read and approved the final manuscript. *ORCID (Open Researcher and Contributor ID) (iD). 


\section{References}

1. Civinni F. Su di um ganglionari rigonfiamento della piñata del piede. Mem Chir Archiespedale Pistoia 1835;4:4-17.

2. Durlacher L. A treatise on corns, bunions, the disease of nails, and the general management of the feet. London: Simpkin, Marshall; 1845.

3. Coughlin MJ, Pinsonneault T. Operative treatment of interdigital neuroma. A long-term follow-up study. J Bone Joint Surg Am. 2001;83(9):1321-8.

4. Morton TG. A peculiar and painful affection of the fourth metatarso-phalengeal articulation. Am J Med Sci 1876;71:37-45.

5. Pastides P, El-Sallakh S, Charalambides C. Morton's neuroma: A clinical versus radiological diagnosis. Foot Ankle Surg. 2012; 18(1):22-4.

6. Valente $M$, Crucil M, Alecci V. Operative treatment of interdigital Morton's neuroma. Chir Organi Mov. 2008;92(1):39-43.

7. Wu KK. Morton's interdigital neuroma: a clinical review of its etiology, treatment, and results. J Foot Ankle Surg. 1996;35(2):112-9.

8. Zanetti M, Strehle JK, Kundert HP, Zollinger H, Hodler J. Morton neuroma: effect of MR imaging findings on diagnostic thinking and therapeutic decisions. Radiology. 1999;213(2):583-8.

9. Gurdezi S, White T, Ramesh P. Alcohol injection for Morton's neuroma: a five-year follow-up. Foot Ankle Int. 2013;34(8):1064-7.

10. Ha'Eri GB, Fornasier VL, Schatzker J. Morton's neuroma--pathogenesis and ultrastructure. Clin Orthop Relat Res. 1979;(141): 256-9.

11. Biasca N, Zanetti M, Zollinger $\mathrm{H}$. Outcomes after partial neurectomy of Morton's neuroma related to preoperative case histories, clinical findings, and findings on magnetic resonance imaging scans. Foot Ankle Int. 1999;20(9):568-75.

12. Sobiesk GA, Wertheimer SJ, Schulz R, Dalfovo M. Sonographic evaluation of interdigital neuromas. J Foot Ankle Surg. 1997; 36(5):364-6.
13. Grice J, Marsland D, Smith G, Calder J. Efficacy of Foot and Ankle Corticosteroid Injections. Foot Ankle Int. 2017;38(1):8-13.

14. Makki D, Haddad BZ, Mahmood Z, Shahid MS, Pathak S, Garnham I. Efficacy of corticosteroid injection versus size of plantar interdigital neuroma. Foot Ankle Int. 2012;33(9):722-6.

15. Thomson CE, Gibson JN, Martin D. Interventions for the treatment of Morton's neuroma. Cochrane Database Syst Rev. 2004;2004(3):CD003118.

16. Dereymaeker G, Schroven I, Steenwerckx A, Stuer P. Results of excision of the interdigital nerve in the treatment of Morton's metatarsalgia. Acta Orthop Belg. 1996;62(1):22-5.

17. Valero J, Gallart J, González D, Deus J, Lahoz M. Multiple interdigital neuromas: a retrospective study of 279 feet with 462 neuromas. J Foot Ankle Surg. 2015;54(3):320-2.

18. Benedetti RS, Baxter DE, Davis PF. Clinical results of simultaneous adjacent interdigital neurectomy in the foot. Foot Ankle Int. 1996;17(5):264-8.

19. Cöster MC, Bremander A, Rosengren BE, Magnusson H, Carlsson A, Karlsson MK. Validity, reliability, and responsiveness of the Selfreported Foot and Ankle Score (SEFAS) in forefoot, hindfoot, and ankle disorders. Acta Orthop. 2014;85(2):187-94.

20. Cöster M, Karlsson MK, Nilsson JÅ, Carlsson A. Validity, reliability, and responsiveness of a self-reported foot and ankle score (SEFAS). Acta Orthop. 2012;83(2):197-203.

21. Kasparek M, Schneider W. Surgical treatment of Morton's neuroma: clinical results after open excision. Int Orthop. 2013;37(9):1857-61.

22. Bucknall V, Rutherford D, MacDonald D, Shalaby H, McKinley J, Breusch SJ. Outcomes following excision of Morton's interdigital neuroma: a prospective study. Bone Joint J. 2016;98-B(10):1376-81.

23. Reichert P, Zimmer K, Witkowski J, Wnukiewicz W, Kuliński S, Gosk J. Long-Term Results of Neurectomy Through a Dorsal Approach in the Treatment of Morton's Neuroma. Adv Clin Exp Med. 2016;25(2):295-302. 